\title{
Comparative Evaluation of Apical Sealing Ability of Different Obturation Techniques by Confocal Microscopy: An In Vitro Study
}

\author{
Reetu Arora ${ }^{1} \quad$ Yogesh Kumar $^{1} \quad$ Neetu Jindal ${ }^{1} \quad$ Renu Aggarwal ${ }^{1} \quad$ Kavneet Takhar $^{1}$ \\ ${ }^{1}$ Department of Conservative Dentistry and Endodontics, Surendera \\ Dental College and Research Institute, Sriganganagar, Rajasthan, \\ India \\ Address for correspondence Neetu Jindal, BDS, MDS, Department \\ of Conservative Dentistry and Endodontics, Surendera Dental \\ College and Research Institute, Sriganganagar 335001, Rajasthan, \\ India (e-mail: drneetujindal@gmail.com).
}

Dent J Adv Stud 2020;8:55-59

\begin{abstract}
Introduction The aim of obturation in the root canal is to completely seal the canal space to eliminate all the portals of entry and exit between root canal and periodontal space. Various techniques have been developed to achieve a hermetic seal.

Materials and Methods As many as 150 extracted human maxillary central incisors were taken for the study. Biomechanical preparation was done up to F5 protaper file. According to different obturation techniques, samples were divided into six groups, keeping 30 samples in experimental and 15 samples in control groups. GroupI-LateralCondensation, GroupII-Thermafil, Group III-Beefill, GroupIV-GuttaFlow, Group V-Positive Control group, Group VI-Negative Control group. After obturation, the samples were immersed in $2 \%$ Rhodamine-B dye for 24 hours. Each sample was longitudinally sectioned to examine under confocal laser scanning microscope.

Statistical Analysis The results were evaluated with ANOVA and posthoc Tukey honest significant difference (HSD) comparison test.

Keywords

- apical sealing ability

- guttaflow

- confocal laser

scanning microscope

- microleakage

Results The mean values of dye penetration of different groups were Group I (Lateral Condensation) $\mathbf{1 . 5 1} \pm \mathbf{0 . 4 5 1}$, Group II (Thermafil) $0.918 \pm \mathbf{0 . 3 9 9}$, Group III (Beefill) $1.30 \pm 0.559$. Group IV (GuttaFlow) $0.655 \pm \mathbf{0 . 3 9 6}$, Group V (Positive Control group) $\mathbf{1 . 9 6} \pm \mathbf{0 . 0 4 6}$, Group VI (Negative Control group) $\mathbf{0} \pm \mathbf{0}$. The lowest mean value of apical microleakage was found in GuttaFlow amongst all experimental groups.

Conclusion It can be concluded that the GuttaFlow obturating material exhibited better apical sealing ability with canal walls.
\end{abstract}

\section{Introduction}

One of the goals for successful endodontic procedure is presence of hermetic seal of the root canal system. To ensure long-term success, total obliteration of root canal space and fluid tight seal of the apical area must be followed. This seal acts as a barrier for the microleakage. ${ }^{1}$

Various techniques have developed to achieve the hermetic seal. Lateral condensation of gutta percha is the most widely practiced technique. It is also considered a gold standard technique. But there are some disadvantages, as it does not adapt well to the canal walls and there is presence

DOI https://doi.org/ 10.1055/s-0040-1713579 ISSN 2321-1482. of nonhomogenous mass of gutta percha, which can cause apical microleakage. ${ }^{2}$

To overcome this, various thermoplastic techniques have been developed to increase the plasticity of gutta percha. ${ }^{1}$

Thermafil is a simplified method of obturation technique which consists of plastic or metal carrier coated with $\alpha$ phase gutta percha. It is used with a heating device, thermaprep oven. ${ }^{1}$ It provides a better apical seal, but in this technique, microleakage occurs because of contraction of $\alpha$ phase of gutta percha. ${ }^{3}$

In an attempt to simplify the warm vertical compaction technique, a new device such as BeeFill (VDW, Munich, 
Germany) has been developed. In the Beefill system, gutta percha cartridges heat quickly and the extruder tips can be bent easily. This method fills the root canal using heated gutta percha, but there is no sufficient data available on the apical sealing ability of Beefill. ${ }^{4}$

To overcome the shrinkage of gutta percha in thermoplasticized technique, new materials has been introduced. GuttaFlow is the flowable nonheated gutta percha that does not shrink but expands slightly. Due to the expansion of material, it provides excellent seal. The new root canal filling material GuttaFlow is a modification of a silicon-based sealer, Roekoseal Automix. It also showed excellent adhesion to gutta percha and dentinal wall. ${ }^{5}$

Apical seal is an important factor in determining the success of root canal treatment; therefore, it is necessary to maintain the seal of root canal treatment. Various methods have been introduced to determine the apical microleakage of different techniques by dye penetration methods, radioisotopes, and bacterial penetration. ${ }^{2}$

Thus, the present study is undertaken to evaluate the apical sealing ability of different obturation techniques by confocal microscopy.

\section{Materials and Methods}

One hundred and fifty freshly extracted human maxillary central incisors were selected for the study. All teeth were intact, noncarious and freshly extracted due to periodontal disease or orthodontic purposes. Teeth with root caries, resorption or fracture were not included in the study after preoperative radiograph.

The root surfaces of the sample teeth were cleaned using the scaler tip and kept in hydrogen peroxide to clean the debris and periodontal tissue, if any, for 2 hours.

For standardization of root canal length that was $15 \mathrm{~mm}$, each tooth was decoronated at cemento-enamel junction using water-cooled diamond disc. The working length was determined by placing $\mathrm{K}$ files until it was seen at the apical foramen. The working length of all the samples were determined to be $1 \mathrm{~mm}$ short of apical foramen. After determination of working length, the coronal portion of canal was enlarged by Sx protaper. After using the shaping files, the F1 was selected and passively allowed to move into the canal. When the F1 file achieved the length, the instrument was removed, and the teeth were flushed with $5.25 \% \mathrm{NaOCl}$ and $0.9 \%$ normal saline. Thereafter, finishing of apical area was done with F2 and F3 up to the working length. F4 and F5 (corresponds to no. 50 File) were used in the same manner to enlarge the apical area. Apical patency was maintained throughout the procedure. During instrumentation the teeth were flushed with $5.25 \% \mathrm{NaOCl}$ and $0.9 \%$ normal saline. During cleaning and shaping, 17\% EDTA gel was used as lubricant. According to different obturation techniques, the samples were divided in two control groups $(n=15)$ and four experimental groups $(n=30)$.

\section{Obturation Protocol}

\section{Group I: Lateral Condensation $(\boldsymbol{n}=\mathbf{3 0})$}

Standardized ISO No. 50 gutta percha was trial fitted with tug-back to the working length. It was coated with $\mathrm{AH}$ plus. The master cone was laterally condensed by inserting a finger spreader between it and the root canal wall. The space created by the spreader was filled by gutta percha points. The excess gutta percha was removed with a hot instrument. Obturation was checked radiographically.

\section{Group II: Thermafil $(n=30)$}

Thermafil obturator was selected by using the verifier size 50. Root canal was lightly coated with AH plus sealer. Therma prep oven was preheated before placing the obturators; then, Thermafil obturator was placed in thermaprep oven. After removing the obturator from oven, firm apical pressure was used to insert Thermafil obturator. After radiographic verification, the carrier shaft was removed with a burr that was designed for this purpose. The complete filling of canal was checked radiographically.

\section{Group III: Beefill $(n=30)$}

The canal was coated with AH plus sealer. Then, an apical plugger short of 3 to $5 \mathrm{~mm}$ of working length was penetrated in the canal. The BeeFill system (VDW, Munich, Germany) was set to $180^{\circ} \mathrm{C}$ and at $60 \%$ flow rate. After placing the gutta percha in apical portion, back filling of was done by placing the needle against the apical gutta percha. After compaction, obturation was checked radiographically.

\section{Group IV: GuttaFlow $(n=30)$}

Standardized ISO No. 50 gutta percha master cone was selected. GuttaFlow was applied in the canal with the help of lentulospiral. Master cone was coated with GuttaFlow and placed in the canal. GuttaFlow dispenser was used to fill any additional space left in the canal. Master cone was seared off with the heated instrument. Radiographs were taken to confirm the complete obturation of canal.

\section{Group V: Positive Control $(n=15)$}

The samples were instrumented up to F5 (50 no. file) and kept unobturated.

\section{Group VI: Negative Control $(n=15)$}

The samples were instrumented up to F5 (50 no. file) and kept unobturated.

The coronal access cavities of all samples were sealed with glass ionomer cement (3M ESPE).

The experimental and positive control samples were coated on their external surface with nail varnish except for $2 \mathrm{~mm}$ in the apical region and then allowed to dry overnight. The negative control samples were coated with nail varnish, including the apical region. All the specimens were 
immersed in 2\% Rhodamine-B (Ases Chemical Works, India) dye for 24 hours.

After dye immersion, the samples were washed under running tap water for 5 minutes and allowed to dry for 24 hours. External layer of nail varnish was removed from each sample. Using a diamond disc, each sample was longitudinally sectioned for examination of dye penetration under confocal laser scanning microscope (-Fig. 1) (Nikon's CLSM model AIR. IMTECH, Chandigarh, India).

\section{Statistical Analysis}

The data was subjected to statistical analysis. One-way ANOVA was used for multiple group comparisons followed by posthoc Tukey's honest significant difference (HSD) test for differences among mean.

\section{Results}

The mean values of dye penetration of different groups were $1.51 \pm 0.451$ (Lateral Condensation), $0.918 \pm 0.399$ (Thermafil), $1.30 \pm 0.559$ (Beefill), $0.655 \pm 0.396$ (GuttaFlow), $1.96 \pm 0.046$ (Positive Control group), and $0 \pm 0$ (Negative Control group) (-Table 1). The lowest mean value of apical microleakage was found in GuttaFlow group $0.655 \pm 0.396$. This showed statistically significant difference with lateral condensation and Beefill group $(p<.001)$ ( - Table 2 ). There was no significant difference between Guttaflow and Thermafil $(p>.001)$

(-Table 2).

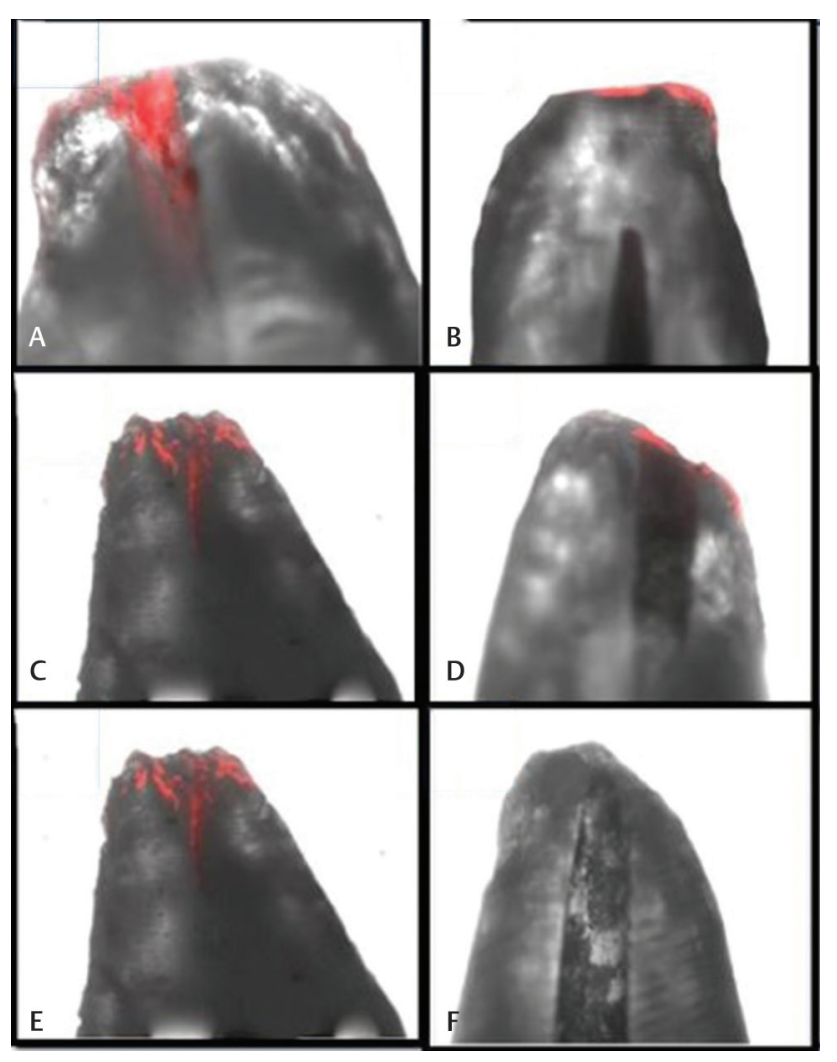

Fig. 1 Confocal images of apical microleakage of samples. (A) Lateral condensation; (B) Thermafil; (C) Beefill; (D) Guttaflow; (E) Positive control; (F) Negative control.
The highest mean value of apical microleakage was found in lateral condensation group $(1.51 \pm 0.451)$ (-Table 2). This was statistically significant with GuttaFlow and Thermafil $(p<.001)$. No statistically significant difference was found between lateral condensation and Beefill ( $p>$.001) (-Table 2 ).

\section{Discussion}

For successful endodontic therapy, three-dimensional (3D) obturation is considered as a -key factor. A 3D obturation is mandatory for a well-defined hermetic seal to prevent microbial leakage, leading to reinfection. ${ }^{2}$

Confocal laser scanning microscope was used to measure apical dye penetration because it does not require special processing of specimens and does not produce artifacts. It produces images with better contrast. ${ }^{6}$

In the present study, the most commonly used and studied technique was lateral condensation because of its easy placement of materials. ${ }^{7}$

Thermafil is a carrier-based, thermoplasticized technique described by Johnson. Johnson claimed that the technique was effective in filling all canal spaces and isthmuses., ${ }^{8,9}$

BeeFill is a thermoplasticized injection device developed to simplify obturation. It is a warm vertical compaction system which consists of backfilling device. ${ }^{4}$

GuttaFlow is a reliable cold filling system for root canals. It contains both gutta percha powder and silicon-based sealer in one form. It is said to have good flowable properties at room temperature and provides better sealing. Due to its property of expansion, instead of shrinkage, it provides better sealing ability. ${ }^{10}$

In the present study, GuttaFlow showed lower mean value of dye penetration $0.655 \pm 0.396$ as compared with other experimental groups; thus, it has better apical sealing ability as compared with other groups. This was according to the results reported by Vyavahare et al. ${ }^{11}$

After GuttaFlow, Gp II (Thermafil) $0.918 \pm 0.399$ showed lesser microleakage as compared with Beefill and lateral condensation groups. This study showed that Thermafil has the lowest mean value of dye penetration than other methods. It may be related to several factors. Thermafil pushes the thermoplasticized gutta percha into the lateral walls of the canal because it acts as a plunger.

There are few studies on comparison of apical sealability of Thermafil and Beefill, but the results of these studies were not in accordance to the present study.

In the present study, Gp II Thermafil showed less dye penetration as compared with Gp III Beefill. There was presence of statistically significant difference between two groups $(p<.001)$. The reason behind it is that Thermafil plastic carrier might act as a plunger which effectively forces the thermoplasticized GP into lateral walls of canal and produces a homogenous mass of gutta percha. ${ }^{2}$ Beefill system is technique-sensitive, and the waiting period to carry heated gutta percha into canal and prevent apical exposure makes it difficult. It requires long-term clinical experience. ${ }^{4}$

Beefill showed lower dye penetration as compared with lateral condensation but statistically nonsignificant difference 
Table 1 Demonstrates the intragroup mean and SD values $(\mathrm{mm})$ of dye penetration after performing different obturating techniques by using one-way ANOVA

\begin{tabular}{|c|c|c|c|c|c|c|}
\hline GROUPS & $\mathrm{N}$ & Mean & SD & F value & $p$-Value & Status \\
\hline GPI (Lateral condensation) & 30 & 1.510 & 0.451 & \multirow[t]{6}{*}{50.53} & \multirow[t]{6}{*}{$<.0001$} & \multirow[t]{6}{*}{ Significant } \\
\hline GP II (Thermafil) & 30 & 0.918 & 0.399 & & & \\
\hline GP III (Beefill) & 30 & 1.304 & 0.559 & & & \\
\hline GP IV (GuttaFlow) & 30 & 0.655 & 0.396 & & & \\
\hline GP V (Positive control) & 30 & 1.961 & 0.046 & & & \\
\hline GP VI (Negative control) & 30 & 0 & 0 & & & \\
\hline
\end{tabular}

Abbreviation: SD, standard deviation.

Table 2 Demonstrates the pairwise intergroup comparison of mean values of dye penetration at apical end after performing different obturating techniques by using posthoc Tukey's HSD test

\begin{tabular}{|l|l|l|l|l|}
\hline Intergroup comparison & $\begin{array}{l}\text { Mean } \\
\text { difference }\end{array}$ & Q value & $p$-Value & Status \\
\hline Lateral condensation vs. Thermafil & 0.592 & 7.9173 & $<.001$ & SIG \\
\hline Lateral condensation vs. Beefill & 0.206 & 2.755 & 0.378 & NS \\
\hline Lateral condensation vs. GuttaFlow & 0.855 & 11.4346 & $<.001$ & SIG \\
\hline Lateral condensation vs. Positive control group & -0.451 & 4.9248 & 0.008 & SIG \\
\hline Lateral condensation vs. Negative control & 1.510 & 16.485 & $<.001$ & SIG \\
\hline Thermafil vs. Beefill & -0.386 & 5.1623 & 0.004 & SIG \\
\hline Thermafil vs Guttaflow & 0.263 & 3.5173 & 0.134 & NS \\
\hline Thermafil vs. Positive control & -1.043 & 11.3892 & $<.001$ & SIG \\
\hline Thermafil vs. Negative control & 0.918 & 10.0206 & $<.001$ & SIG \\
\hline Beefill vs. GuttaFlow & 0.649 & 8.6796 & $<.001$ & SIG \\
\hline Beefill vs. Positive control & -0.657 & 7.1742 & $<.001$ & SIG \\
\hline Beefill vs. Negative control & 1.304 & 14.2356 & $<.001$ & SIG \\
\hline Gutta flow vs. Positive control & -1.306 & 14.2611 & $<.001$ & SIG \\
\hline Gutta flow vs. Negative control & 0.655 & 7.1487 & $<.001$ & SIG \\
\hline Positive control vs. Negative control & 1.961 & 18.5414 & $<.001$ & SIG \\
\hline
\end{tabular}

Abbreviation: HSD, honest significance difference.

was found between these two. The results of Beefill in present study was in accordance with Dadresanfar et al. ${ }^{12}$

Lateral condensation showed more dye penetration values as compared with all other groups. The reason can be this technique does not replicate the canal, as it forms a nonhomogenous mass of gutta percha. ${ }^{13}$

The results of this study showed that GuttaFlow seems to be a promising material and sealer because of its easy handling, and adhesion to gutta percha and dentin. In GuttaFlow, expansion with adhesion creates good apical sealing ability.

However, in clinical conditions, there is presence of dynamic nature of periapical tissue which could be different from the in vitro situation. Further long-term studies are needed for these methods.

\section{Conclusion}

Under the limitations of current in vitro study, the following conclusions can be drawn:
- All experimental groups showed apical dye penetration and none of them were able to achieve hermetic seal.

- GuttaFlow showed minimum apical microleakage, followed by Thermafil, Beefill and lateral condensation. Although there was difference in mean values but GuttaFlow did not show statistically significant difference from Thermafil.

\section{Conflict of Interest}

None declared.

\section{References}

1 Hwang HK, Park HS, Lee YJ. Comparative study on the apical sealing ability according to the obturation techniques. J Korean Acad Conserv Dent 2002;27(3):290-298

2 Emmanuel S, Shantaram K, Sushil KC, Manoj L. An in-vitro evaluation and comparison of apical sealing ability of three different obturation technique - lateral condensation, Obtura II, and Thermafil. J Int Oral Health 2013;5(2):35-43 
3 Priyank H, Mahalakshmi V, Verma A. Comparative analysis on apical microleakage in root canals obturated with Gutta flow, Thermafil and regular Guttapercha with lateral condensation - A stereomicroscopic study. J Adv Med Dent Sci Res 2017;5(10):1-6

4 Yilmaz Z, Deniz D, Özcelik B, et al. Sealing efficiency of BeeFill 2in1 and System B/Obtura II versus single-cone and cold lateral compaction techniques. Oral Surg Oral Med Oral Pathol Oral Radiol Endod 2009;108(6):e51-e55

5 Varun K, Harpreet S, Rajinder B, Samrity P. Qualitative and quantitative comparative evaluation of sealing ability of guttaflow, thermoplasticized gutta percha and lateral compaction for root canal obturation: a cohort, controlled, ex-vivo study. Oral Health Dent Manag 2013;12(3):155-161

6 Chandra SS, Shankar P, Indira R. Depth of penetration of four resin sealers into radicular dentinal tubules: a confocal microscopic study. J Endod 2012;38(10):1412-1416

7 Anantula K, Ganta AK. Evaluation and comparison of sealing ability of three different obturation techniques - Lateral condensation, Obtura II, and GuttaFlow: An in vitro study. J Conserv Dent 2011;14(1):57-61

8 Nair R, Rajaraman G, Gera T, Jeyapreetha P, Nair A. Apical seal comparison of thermoplasticized gutta percha technique and cold lateral condensation technique. Indian J Dent Res 2012;24(4):518-520

9 Ravanshad S, Khayat A. An in-vitro evaluation of apical seal ability of Thermafil obturation versus lateral condensation. J Dent TUMS 2004;1:48-55

10 El Sayed MA, Taleb AA, Balbahaith MS. Sealing ability of three single-cone obturation systems: An in-vitro glucose leakage study. J Conserv Dent 2013;16(6):489-493

11 Vyavahare NK, Baranwal AK, Paul ML. An in-vitro evaluation and correlation of homogeneity, adaptation and microleakage of a silicone based root canal filling material guttaflow: a stereomicroscopic study. Int J Curr Res 2015;7(10):21357-21362

12 Dadresanfar B, Khalilak Z, Shiekholeslami M, Afshar S. Comparative study of the sealing ability of the lateral condensation technique and the BeeFill system after canal preparation by the Mtwo NiTi rotary system. J Oral Sci 2010;52(2):281-285

13 Inan $\mathrm{U}$, Aydemir H, Taşdemir T. Leakage evaluation of three different root canal obturation techniques using electrochemical evaluation and dye penetration evaluation methods. Aust Endod J 2007;33(1):18-22 